\title{
SUFFICIENT CONDITIONS FOR ROBUST OBSERVABILITY OF DISCRETE LINEAR TIME-VARYING SYSTEMS
}

\author{
Jaewon Seo", Dohyoung Chung ${ }^{* *}$, Chan Gook Park ${ }^{* * *}$, Jang Gyu Lee* \\ * School of Electrical Engineering and Computer Science, \\ Seoul National University, Seoul, 151-744, Korea \\ ** Samsung Electronics Corp., Ltd., Suwon 442-742, Korea \\ *** School of Mechanical and Aerospace Engineering, \\ Seoul National University, Seoul, 151-744, Korea
}

\begin{abstract}
The observability of discrete linear time-varying (LTV) systems with normbounded parameter uncertainties is analysed in this paper. Like in continuous case (Chung, et al., 1999), the sufficient conditions, which ensure the observability of the uncertain system, are proposed. In discrete cases, reconstructibility is no longer equivalent to observability, which results in the different form of equation in the observability conditions. The proposed conditions allow the evaluation of the observability for the discrete LTV systems with norm-bounded parameter uncertainty. Copyright C 2005 IFAC
\end{abstract}

Keywords: Robustness, Observability, Time-varying systems, Discrete-time systems, Uncertain linear systems

\section{INTRODUCTION}

Previous studies on the robust stabilization of continuous linear systems with norm-bounded parameter uncertainties have also extended into the realm of discrete linear systems (De Souza, et al., 1993; Garcia, et al., 1994; Xie, et al., 1991; Xie, et al., 1994). For the discrete cases, somewhat different robustness results are obtained due to the difference in the nature and the structure between continuous linear systems and discrete linear systems.

The study on the robustness of observability for continuous linear time-varying systems (Chung, et $a l ., 1999)$ can also be extended for the discrete linear time-varying systems. Unlike the continuous cases, the observability and the reconstructibility are no longer equivalent for discrete linear time-varying systems. Therefore it is expected that the conditions that ensure the observability in discrete cases are different from those of Chung, et al. (1999).

As related study, Moheimani, et al. (1996) proposed necessary and sufficient conditions for the robust observability of discrete systems. Although the systems and assumptions on uncertainties in Moheimani, et al. (1996) are similar to current paper, it seems that the definition of the robust observability and the results proposed are quite different.

In this paper, sufficient conditions for observability of discrete linear time-varying systems with normbounded parameter uncertainty are studied. The formulation of the norm-bounded parameter uncertainty for discrete linear time-varying systems is similar to that of the continuous case. Because of the inherent differences between continuous linear systems and discrete linear systems, different results for the robustness of observability are derived.

\section{PRELIMINARIES}

Consider the discrete LTV system.

$$
\begin{aligned}
x(k+1) & =A(k) x(k)+B(k) u(k) \\
y(k) & =C(k) x(k), x\left(k_{0}\right)=x_{0}
\end{aligned}
$$

where $A(k), B(k)$, and $C(k)$ are $n \times n, n \times p$, and $m \times n$ matrices, respectively. The each element of 
$A(k), B(k)$, and $C(k)$ is assumed to be a real and bounded function of $k . x(k), u(k)$, and $y(k)$ are the state variable vector $(n \times 1)$, the input vector $(p \times 1)$, and the output vector $(m \times 1)$ of the system, respectively. If $\Phi\left(k, k_{0}\right)$ is the transition matrix for $x(k+1)=A(k) x(k)$, then the solution of the linear time-varying system (1) is given by,

$$
\begin{aligned}
& x(k)=\Phi\left(k, k_{0}\right) x\left(k_{0}\right)+\sum_{i=k_{0}}^{k-1} \Phi(k, i+1) B(i) u(i) \\
& k>k_{0} .
\end{aligned}
$$

The observability of LTV systems represents the ability to determine the state variable using the input and output of the system. It can be described using the concept of reconstructibility. The state $x_{1}$ at $k_{1}$ is said to be unreconstructible on $\left[k_{0}, k_{1}\right]$ if and only if the zero input response for the LTV system (1), which corresponds to the state $x_{1}$ at $k_{1}$, is zero on $\left[k_{0}, k_{1}\right]$. The discrete linear time-varying system (1) is reconstructible on $\left[k_{0}, k_{1}\right]$ if and only if the zero state is the only state that is unreconstructible on $\left[k_{0}, k_{1}\right]$ (Callier and Desoer, 1991). Unlike the continuous cases, the observability and the reconstructibility are no longer equivalent for discrete linear time-varying systems unless $A(k)$ in (1) is nonsingular for all $k$. Since the condition that $A(k)$ is nonsingular is too strong, we will not use the concept of reconstructibility and only reside in the concept of observability.

Consider the following linear map $\mathfrak{J}_{O}$ from $\mathfrak{R}^{n}$ to $l_{2}^{m}\left[k_{0}, k_{1}\right]$;

$$
\begin{aligned}
& \mathfrak{I}_{O}(x)(k)=C(k) \Phi\left(k, k_{0}\right) x, x \in \mathfrak{R}^{n} \\
& k \in\left[k_{0}, k_{1}\right]
\end{aligned}
$$

$\mathfrak{I}_{O}$ is referred as the observability map. The LTV system (1) is observable on $\left[k_{0}, k_{1}\right]$ if and only if $\mathfrak{I}_{O}$ is injective, which means $\mathfrak{I}_{O}$ is a one-to-one map.

In the following sections, the robustness of observability for discrete LTV systems under the presence of norm-bounded parameter uncertainty is studied using these concepts.

\section{THE ROBUSTNESS OF OBSERVABILITY}

The robustness of observability for discrete linear time-varying systems can be obtained in similar manners to that of continuous linear time-varying systems (Chung, et al., 1999). In this section a discrete linear time-varying system is considered with norm-bounded parameter uncertainty in its system matrix such as,

$$
\begin{aligned}
& x(k+1)=(A(k)+H(k) F(k) E(k)) x(k) \\
& y(k)=C(k) x(k)
\end{aligned}
$$

where $H(k)$ and $E(k)$ are real matrices with appropriate dimensions. It is assumed that $F(\cdot)$ is the actual norm-bounded parameter uncertainty such as,

$$
F^{T}(k) F(k) \leq \rho^{2} I, \forall k
$$

In order to study the robustness of observability for the discrete linear time-varying system (4), consider the linear map $\mathfrak{I}_{O}$ from $\mathfrak{R}^{n}$ to $l_{2}^{m}\left[k_{0}, k_{1}\right]$, such as,

$$
\begin{aligned}
& \mathfrak{J}_{O}(x)(k)=C(k) \Phi_{F}\left(k, k_{0}\right) x \\
& x \in \mathfrak{R}^{n}, k \in\left[k_{0}, k_{1}\right]
\end{aligned}
$$

where $\Phi_{F}(k, j)$ is the state transition matrix for the system matrix $A(k)+H(k) F(k) E(k)$. The discrete linear time-varying system (4) is observable on $\left[k_{0}, k_{1}\right]$ if and only if the null space of the linear map $\mathfrak{I}_{O}$ from $\mathfrak{R}^{n}$ to $l_{2}^{m}\left[k_{0}, k_{1}\right]$ is only the zero state. The following lemma is used to obtain the conditions on the robustness of observability for discrete linear time-varying systems.

Lemma 1: The state transition matrix $\Phi_{F}(k, j)$ for the system matrix $A(k)+H(k) F(k) E(k)$ in (4), where $F(k)$ is an arbitrary norm-bounded parameter uncertainty, can be expressed as follows.

$$
\begin{aligned}
& \Phi_{F}(k, j) \\
& =\left\{\begin{array}{c}
I, \text { if } k=j, \\
\Phi(k, j)+\sum_{i=j}^{k-1} \Phi(k, i+1) H(i) F(i) E(i) \Phi_{F}(i, j), \\
\text { if } k>j,
\end{array}\right.
\end{aligned}
$$

where $\Phi(k, j)$ is the state transition matrix for the system matrix $A(k)$.

Proof: It is proved by the mathematical induction. Suppose that $k=j+1$. Using (7), $\Phi_{F}(j+1, j)$ becomes,

$$
\Phi_{F}(j+1, j)=A(j)+H(j) F(j) E(j)
$$

Hence (7) holds for $k=j+1$. Assume that (7) holds, then

$$
\begin{aligned}
& \Phi(k+1, j)+\sum_{i=j}^{k} \Phi(k+1, i+1) H(i) F(i) E(i) \Phi_{F}(i, j) \\
& =A(k) \Phi(k, j)+\sum_{i=j}^{k-1} \Phi(k+1, i+1) H(i) F(i) E(i) \Phi_{F}(i, j) \\
& +H(k) F(k) E(k) \Phi_{F}(k, j) \\
& =A(k) \Phi_{F}(k, j)+H(k) F(k) E(k) \Phi_{F}(k, j) \\
& =\Phi_{F}(k+1, j)
\end{aligned}
$$


which implies that (7) holds for $k$ replaced by $k+1$.

Q.E.D.

Based on the lemma, the following theorem provides the sufficient condition for the robustness of observability for discrete linear time-varying systems.

Theorem 1: Suppose that the linear time-varying system (4) is observable on $\left[k_{0}, k_{1}\right]$ when $F(k)=0$, $\forall k \in\left[k_{0}, k_{1}\right]$. Then it is observable on $\left[k_{0}, k_{1}\right]$ for all norm-bounded parameter uncertainty that satisfy (5) if the condition (10) holds.

$$
\begin{aligned}
& \left\|\sum_{i=k_{0}}^{k_{1}-1}\left(\begin{array}{l}
\left.W_{O}^{-1}\left[k_{0}, k_{1}\right] \Phi^{T}\left(i+1, k_{0}\right) W_{O}\left[i+1, k_{1}\right]\right) \\
H(i) E(i) \Phi_{F}\left(i, k_{0}\right)
\end{array}\right)\right\|_{i 2}<1 \\
& \forall F^{T}(k) F(k) \leq \rho^{2} I, k \in\left[k_{0}, k_{1}-1\right],
\end{aligned}
$$

where \|\|$_{i 2}$ is the induced 2-norm. $\Phi_{F}(\cdot$,$) and \Phi(\cdot, \cdot)$ are defined in the lemma 1 and $W_{O}\left[k_{0}, k_{1}\right]$ is defined as

$$
W_{O}\left[k_{0}, k_{1}\right] \equiv \sum_{i=k_{0}}^{k_{1}} \Phi^{T}\left(i, k_{0}\right) C^{T}(i) C(i) \Phi\left(i, k_{0}\right)
$$

Proof: For an arbitrary nonzero $x_{0} \in \mathfrak{R}^{n}$ and an arbitrary norm-bounded parameter uncertainty $F(k)$, consider the following linear map on $\left[k_{0}, k_{1}\right]$,

$$
\mathfrak{I}_{F}\left(x_{0}\right)(k) \equiv C(k) \Phi_{F}\left(k, k_{0}\right) x_{0}, k \in\left[k_{0}, k_{1}\right]
$$

Consider the following matrix summation,

$$
\sum_{i=k_{0}}^{k_{1}} \Phi^{T}\left(i, k_{0}\right) C^{T}(i) \mathfrak{I}_{F}\left(x_{0}\right)(i)
$$

Then using lemma 1, it becomes

$$
\begin{aligned}
& \sum_{i=k_{0}}^{k_{1}} \Phi^{T}\left(i, k_{0}\right) C^{T}(i) \mathfrak{I}_{F}\left(x_{0}\right)(i) \\
& =\sum_{i=k_{0}}^{k_{1}} \Phi^{T}\left(i, k_{0}\right) C^{T}(i) C(i) \Phi\left(i, k_{0}\right) x_{0} \\
& +\sum_{i=k_{0}+1}^{k_{1}} \sum_{j=k_{0}}^{i-1}\left(\begin{array}{l}
\Phi^{T}\left(i, k_{0}\right) C^{T}(i) C(i) \Phi(i, j+1) \\
\cdot H(j) F(j) E(j) \Phi_{F}\left(j, k_{0}\right) x_{0}
\end{array}\right)
\end{aligned}
$$

(13) can be expressed as

$$
\begin{aligned}
& \sum_{i=k_{0}}^{k_{1}} \Phi^{T}\left(i, k_{0}\right) C^{T}(i) \mathfrak{J}_{F}\left(x_{0}\right)(i) \\
& =\sum_{i=k_{0}}^{k_{1}} \Phi^{T}\left(i, k_{0}\right) C^{T}(i) C(i) \Phi\left(i, k_{0}\right) x_{0} \\
& +\sum_{j=k_{0}}^{k_{1}-1} \Phi^{T}\left(j+1, k_{0}\right) \\
& \cdot \sum_{i=j+1}^{k_{1}}\left(\Phi^{T}(i, j+1) C^{T}(i) C(i) \Phi(i, j+1)\right. \\
&
\end{aligned}
$$

Using $W_{O}\left[k_{0}, k_{1}\right],(14)$ can be expressed as

$$
\begin{aligned}
& \sum_{i=k_{0}}^{k_{1}} \Phi^{T}\left(i, k_{0}\right) C^{T}(i) \mathfrak{J}_{F}\left(x_{0}\right)(i)=W_{O}\left[k_{0}, k_{1}\right] x_{0} \\
& +\sum_{j=k_{0}}^{k_{1}-1}\left[\begin{array}{l}
\Phi^{T}\left(j+1, k_{0}\right) W_{O}\left[j+1, k_{1}\right] H(j) \\
\cdot F(j) E(j) \Phi_{F}\left(j, k_{0}\right) x_{0}
\end{array}\right]
\end{aligned}
$$

Since (4) is observable on $\left[k_{0}, k_{1}\right]$ when $F(k)=0$ for $k \in\left[k_{0}, k_{1}\right], W_{o}\left[k_{0}, k_{1}\right]$ is nonsingular. Thus (15) can be rewritten by,

$$
\begin{aligned}
& \sum_{i=k_{0}}^{k_{1}} W_{O}^{-1}\left[k_{0}, k_{1}\right] \Phi^{T}\left(i, k_{0}\right) C^{T}(i) \mathfrak{I}_{F}\left(x_{0}\right)(i)= \\
& {\left[I+\sum_{j=k_{0}}^{k_{1}-1}\left(\begin{array}{l}
W_{O}^{-1}\left[k_{0}, k_{1}\right] \Phi^{T}\left(j+1, k_{0}\right) W_{O}\left[j+1, k_{1}\right] \\
\cdot H(j) F(j) E(j) \Phi_{F}\left(j, k_{0}\right)
\end{array}\right)\right] x_{0}}
\end{aligned}
$$

Suppose that the linear time-varying system (4) is unobservable for some norm-bounded parameter uncertainty $F_{1}(\cdot)$ that satisfies (5). Then there must exist nonzero vector state $x_{1} \in \mathfrak{R}^{n}$ such that

$$
\mathfrak{I}_{F_{1}}\left(x_{1}\right)(k)=0, \forall k \in\left[k_{0}, k_{1}\right] \text {. }
$$

Using (16) and (17), the following relation must hold.

$$
\left[I+\sum_{j=k_{0}}^{k_{1}-1}\left(\begin{array}{l}
W_{O}^{-1}\left[k_{0}, k_{1}\right] \Phi^{T}\left(j+1, k_{0}\right) W_{O}\left[j+1, k_{1}\right] \\
\cdot H F_{1}(j) E \Phi_{F_{1}}\left(j, k_{0}\right)
\end{array}\right)\right] x_{1}=0
$$

The relation (18) contradicts the condition (10).

Q.E.D.

Theorem 1 provides the sufficient conditions that keep $\mathfrak{I}_{F}(\cdot)$ injective. It can be further extended by the following theorem.

Theorem 2: Suppose that $W_{F}\left(k_{0}, k_{1}\right)$ and $W_{E}\left(k_{0}, k_{1}\right)$ satisfy the conditions (19) for some positive $\alpha$ and $\beta$. If $\alpha \beta<1$, then the sufficient condition (10) holds.

$$
\begin{aligned}
& W_{F}\left(k_{0}, k_{1}\right) \leq \alpha^{2} I, \\
& \forall F^{T}(k) F(k) \leq \rho^{2} I, k \in\left[k_{0}, k_{1}-1\right] \\
& W_{E}\left(k_{0}, k_{1}\right) \leq \beta^{2} I
\end{aligned}
$$

where $W_{F}\left(k_{0}, k_{1}\right)$ and $W_{E}\left(k_{0}, k_{1}\right)$ are defined by,

$$
\begin{aligned}
& W_{F}\left(k_{0}, k_{1}\right) \equiv \sum_{i=k_{0}}^{k_{1}-1} \Phi_{F}^{T}\left(i, k_{0}\right) E^{T}(i) F^{T}(i) F(i) E(i) \Phi_{F}\left(i, k_{0}\right) \\
& W_{E}\left(k_{0}, k_{1}\right) \equiv \\
& \sum_{i=k_{0}}^{k_{1}-1}\left(\begin{array}{l}
W_{O}^{-1}\left[k_{0}, k_{1}\right] \Phi^{T}\left(i+1, k_{0}\right) W_{O}\left[i+1, k_{1}\right] H(i) \\
H^{T}(i) W_{O}\left[i+1, k_{1}\right] \Phi\left(i+1, k_{0}\right) W_{O}^{-1}\left[k_{0}, k_{1}\right]
\end{array}\right)
\end{aligned}
$$


Proof: Suppose that for an arbitrary nonzero $x_{0} \in \mathfrak{R}^{n}$, define $u(k)$, such as,

$$
u(k) \equiv F(k) E(k) \Phi_{F}\left(k, k_{0}\right) x_{0}, k \in\left[k_{0}, k_{1}-1\right]
$$

From (19) and (20), the following must hold.

$$
\|u\|_{2}^{2}=x_{0}^{T} W_{F}\left(t_{0}, t_{1}\right) x_{0} \leq \alpha^{2}\left|x_{0}\right|_{2}^{2}
$$

Using (21), assume the following.

$$
y_{0}=\sum_{i=k_{0}}^{k_{1}-1} W_{O}^{-1}\left[k_{0}, k_{1}\right] \Phi^{T}\left(i+1, k_{0}\right) W_{O}\left[i+1, k_{1}\right] H(i) u(i)
$$

By the property of discrete linear operator's induced norm, the following can be obtained from (22).

$$
\left|y_{0}\right|_{2}^{2} \leq \lambda_{M}\left(W_{E}\left(t_{0}, t_{1}\right)\right)\|u\|_{2}^{2} \leq \beta^{2}\|u\|_{2}^{2}
$$

Combining (22) and (23), the following can be derived.

$$
\left|y_{0}\right|_{2}^{2} \leq \alpha^{2} \beta^{2}\left|x_{0}\right|_{2}^{2}
$$

Since (24) holds for an arbitrary nonzero $x_{0} \in \mathfrak{R}^{n}$ and $\alpha \beta<1$, the sufficient condition (10) in theorem 1 holds.

Q.E.D.

The computation of the upper bound matrix for $W_{F}\left(k_{0}, k_{1}\right)$ is shown in the following theorem.

Theorem 3: Suppose that the linear time-varying system (4) is observable on $\left[k_{0}, k_{1}\right]$ when $F(k)=0$, $\forall k \in\left[k_{0}, k_{1}\right]$. Then it is observable on $\left[k_{0}, k_{1}\right]$ for all norm-bounded parameter uncertainty that satisfy (5) if the following conditions hold for a number sequence $\varepsilon(k)$.

$$
\begin{aligned}
& \rho \lambda_{M}^{1 / 2}\left(W_{1}\left(k_{0}\right)\right) \lambda_{M}^{1 / 2}\left(W_{3}^{-1}\left(k_{0}\right) W_{2}\left(k_{0}\right) W_{3}^{-1}\left(k_{0}\right)\right)<1 \\
& \varepsilon(k) I>H^{T}(k) W_{1}(k+1) H(k), \\
& \forall k=k_{1}-1, k_{1}-2, \cdots, k_{0}
\end{aligned}
$$

where $W_{1}(\cdot), W_{2}(\cdot)$ and $W_{3}(\cdot)$ are the solutions of the following matrix difference equations.

$$
\begin{aligned}
& W_{1}(k)=A^{T}(k)\left[W_{1}(k+1)+W_{1}(k+1) H(k)\right. \\
& \cdot\left[\varepsilon(k) I-H^{T}(k) W_{1}(k+1) H(k)\right]^{-1} \\
& \left.\cdot H^{T}(k) W_{1}(k+1)\right] A(k)+\left(1+\rho^{2} \varepsilon(k)\right) E^{T}(k) E(k), \\
& W_{1}\left(k_{1}\right)=0 \\
& W_{2}(k)=A^{T}(k)\left[W_{2}(k+1)\right. \\
& \left.+W_{3}(k+1) H(k) H^{T}(k) W_{3}(k+1)\right] A(k), \\
& W_{2}\left(k_{1}\right)=0
\end{aligned}
$$

$$
\begin{aligned}
& W_{3}(k)=A^{T}(k) W_{3}(k+1) A(k)+C^{T}(k) C(k), \\
& W_{3}\left(k_{1}+1\right)=0
\end{aligned}
$$

Proof: For an arbitrary parameter uncertainty $F_{0}(\cdot)$ that satisfies $(5)$, define $\bar{W}_{1}(\cdot)$

$$
\bar{W}_{1}(k) \equiv \sum_{i=k}^{k_{1}-1} \Phi_{F_{0}}^{T}(i, k) E^{T}(i) E(i) \Phi_{F_{0}}(i, k)
$$

Then the following must be satisfied.

$$
W_{F_{0}}\left(k_{0}, k_{1}\right) \leq \rho^{2} \bar{W}_{1}\left(k_{0}\right)
$$

where $W_{F_{0}}$ is defined in (20). $\bar{W}_{1}\left(k_{0}\right)$ can be expressed as the solution of the following matrix difference equation.

$$
\begin{aligned}
& \overline{W_{1}}(k)=\left[A(k)+H(k) F_{0}(k) E(k)\right]^{T} \\
& \cdot \overline{W_{1}}(k+1)\left[A(k)+H(k) F_{0}(k) E(k)\right]+E^{T}(k) E(k), \\
& \bar{W}_{1}\left(k_{1}\right)=0 .
\end{aligned}
$$

Since $W_{1}(k+1)$ in $(27)$ is a symmetric positive semidefinite matrix, there always exists $W_{1}^{1 / 2}(k+1)$ which is also a symmetric positive semi-definite matrix. The following equation holds for the matrix differential equation (27).

$$
\begin{aligned}
& A^{T}(k) W_{1}(k+1) H(k) F_{0}(k) E(k) \\
& +E^{T}(k) F_{0}^{T}(k) H^{T}(k) W_{1}(k+1) A(k) \\
& +E^{T}(k) F_{0}^{T}(k) H^{T}(k) W_{1}(k+1) H(k) F_{0}(k) E(k) \\
& \leq A^{T}(k) W_{1}(k+1) H(k)[\varepsilon(k) I \\
& \left.-H^{T}(k) W_{1}(k+1) H(k)\right]^{-1} H^{T}(k) W_{1}(k+1) A(k) \\
& +\rho^{2} \varepsilon(k) E^{T}(k) E(k)
\end{aligned}
$$

Since the inequality (33) holds for arbitrary normbounded parameter uncertainty $F(\cdot)$ that satisfies (5), the matrix difference equation (27) can be expressed as follows.

$$
\begin{aligned}
& W_{1}(k)=\left[A(k)+H(k) F_{0}(k) E(k)\right]^{T} \\
& \cdot W_{1}(k+1)\left[A(k)+H(k) F_{0}(k) E(k)\right] \\
& +E^{T}(k) E(k)+\widetilde{W}_{1}(k), \\
& W_{1}\left(k_{1}\right)=0
\end{aligned}
$$

where $\tilde{W}_{1}(k) \geq 0, \quad k=k_{1}-1, k_{1}-2, \cdots, k_{0}$. Define $\Delta W_{1}(k)=W_{1}(k)-\bar{W}_{1}(k)$, then the matrix difference equation $\Delta W_{1}(k)$ becomes,

$$
\begin{aligned}
& \Delta W_{1}(k)=\left[A(k)+H(k) F_{0}(k) E(k)\right] \Delta W_{1}(k+1) \\
& \cdot\left[A^{T}(k)+E^{T}(k) F_{0}^{T}(k) H^{T}(k)\right]+\widetilde{W}_{1}(k), \\
& \Delta W_{1}\left(k_{1}\right)=0
\end{aligned}
$$


Hence $\quad \Delta W_{1}\left(k_{0}\right) \geq 0$. For all norm-bounded parameter uncertainty that satisfy (5), the following is satisfied.

$$
W_{F}\left(k_{0}, k_{1}\right) \leq \rho^{2} W_{1}\left(k_{0}\right)
$$

Furthermore it can be shown that $W_{E}\left(k_{0}, k_{1}\right)$ can be expressed as,

$$
W_{E}\left(k_{0}, k_{1}\right)=W_{3}^{-1}\left(k_{0}\right) W_{2}\left(k_{0}\right) W_{3}^{-1}\left(k_{0}\right)
$$

If the conditions (25), (26) are satisfied, the sufficient conditions in theorem 2 are satisfied.

$$
\text { Q.E.D. }
$$

The number sequence $\varepsilon(k)$ can be arbitrarily chosen as long as it satisfies (26). Note that when $W_{1}(k+1)$ becomes nonsingular, more compact form of the difference equation is available, which can be obtained by applying the matrix inversion lemma to the matrix difference equation (27).

$$
\begin{aligned}
& W_{1}(k)=A^{T}(k)\left[W_{1}^{-1}(k+1)\right. \\
& \left.-\frac{1}{\varepsilon(k)} H(k) H^{T}(k)\right]^{-1} A(k) \\
& +\left(\rho^{2} \varepsilon(k)+1\right) E^{T}(k) E(k)
\end{aligned}
$$

Note that these matrix difference equations are backward equations. The forward equations for the robustness of observability for general discrete linear time-varying systems cannot be obtained using the concept of reconstructibility because, as previously mentioned, reconstructibility is no longer equivalent to observability in discrete cases unless the system matrices are always nonsingular. In order to apply the concept of reconstructibility, the system matrix $A(k)+H(k) F(k) E(k)$ must be nonsingular for all $k$ and for all norm-bounded uncertainties that satisfy (5) under the given norm bound. Hence the forward equations for the robustness of observability are relevant only for a specific kind of system, only when the nominal system matrix is nonsingular and the norm-bounded parameter uncertainties do not violate the nonsingularity of the system matrix. Since the forward equations have limited usefulness, only backward equations are considered in this paper.

\section{EXAMPLE}

Consider the following discrete linear time-varying uncertain system.

$$
\begin{aligned}
x(k+1) & =\left[\begin{array}{cc}
0 & 1+f(k) \\
0 & 1
\end{array}\right] x(k) \\
& =\left(\left[\begin{array}{ll}
0 & 1 \\
0 & 1
\end{array}\right]+\left[\begin{array}{l}
1 \\
0
\end{array}\right] f(k)\left[\begin{array}{ll}
0 & 1
\end{array}\right]\right) x(k) \\
y(k) & =\left[\begin{array}{ll}
1 & 0
\end{array}\right] x(k)
\end{aligned}
$$

where the uncertainty, $f(k)$, is assumed to satisfy the following condition,

$$
f(k)^{2} \leq 1, \forall k
$$

For the system (39) with the uncertainty constraint (40), state transition matrices of nominal system matrix $A(k)$ and uncertain system matrix $A(k)+H(k) F(k) E(k)$ are respectively as follows:

$$
\begin{aligned}
& \Phi(k, j)=\left[\begin{array}{ll}
0 & 1 \\
0 & 1
\end{array}\right], \\
& \Phi_{F}(k, j)=\left[\begin{array}{cc}
0 & 1+f(k-1) \\
0 & 1
\end{array}\right]
\end{aligned}
$$

where $k, j \in\left[k_{0}, k_{1}\right]$.

For evaluation of robust observability, theorem 2 can be applied. To apply the theorem $2, W_{F}\left(k_{0}, k_{1}\right)$ and $W_{E}\left(k_{0}, k_{1}\right)$ in $(20)$ and $W_{o}\left[k_{0}, k_{1}\right]$ in theorem 1 must be calculated and the results are given by:

$$
\begin{aligned}
& W_{o}\left[k_{0}, k_{1}\right]=\sum_{i=k_{0}}^{k_{1}} \Phi^{T}\left(i, k_{0}\right) C^{T}(i) C(i) \Phi\left(i, k_{0}\right) \\
& =\left[\begin{array}{cc}
1 & 0 \\
0 & k_{1}-k_{0}
\end{array}\right] \\
& W_{F}\left(k_{0}, k_{1}\right)=\sum_{i=k_{0}}^{k_{1}-1} \Phi_{F}^{T}\left(i, k_{0}\right) E^{T}(i) F^{T}(i) F(i) E(i) \Phi_{F}\left(i, k_{0}\right) \\
& =\left[\begin{array}{cc}
0 & 0 \\
0 & \sum_{k=k_{0}}^{k_{1}-1} f(k)^{2}
\end{array}\right] \\
& W_{E}\left(k_{0}, k_{1}\right)=\sum_{i=k_{0}}^{k_{1}-1}\left(\begin{array}{l}
W_{O}^{-1}\left[k_{0}, k_{1}\right] \Phi^{T}\left(i+1, k_{0}\right) W_{O}\left[i+1, k_{1}\right] H(i) \\
\cdot H^{T}(i) W_{O}\left[i+1, k_{1}\right] \Phi\left(i+1, k_{0}\right) W_{O}^{-1}\left[k_{0}, k_{1}\right]
\end{array}\right) \\
& =\left[\begin{array}{cc}
0 & 0 \\
0 & \frac{1}{k_{1}-k_{0}}
\end{array}\right]
\end{aligned}
$$

According to theorem 2, if the following inequality (42) satisfies, the uncertain system (39) is observable on $\left[k_{0}, k_{1}\right]$ for all uncertainties that satisfy (40).

$$
\frac{1}{k_{1}-k_{0}}\left(f\left(k_{0}\right)^{2}+\cdots+f\left(k_{1}-1\right)^{2}\right)<1
$$

The left side of the inequality (42) is the average value of $f\left(k_{i}\right)^{2}$ 's in the interval. Considering that the system (39) becomes unobservable if $f(k)=-1$ for all $k$, this result is intuitively understandable.

\section{CONCLUSION}

The sufficient conditions guaranteeing the robust observability are proposed for discrete linear timevarying systems with norm-bounded parameter uncertainty. 
Using the state transition matrix relationship between the nominal system and the uncertain system, the sufficient conditions which guarantee the observability under the norm-bounded uncertainty are derived. The observability grammian of the nominal system is also used in the derivation. It is proved that if the solutions of certain matrix difference equations satisfy the sufficient conditions, the robustness of observability can be established. Under the sufficient conditions, the observability map from the state to output space remains injective. For discrete cases, in the derivation of the sufficient conditions, the observability grammian is used instead of the reconstructibility grammian and the obtained matrix difference equations are in backward form while in continuous case they are in forward form.

\section{ACKNOWLEDGEMENT}

The authors would like to acknowledge the support and assistance of ACRC in SNU and BK-21 project in Korea.

\section{REFERENCES}

Callier, F.M. and C.A. Desoer (1991). Linear System Theory. Springer-Verlag, New York.

Chung, D., C.G. Park and J.G. Lee (1999). The Robustness of Controllability and Observability for Continuous Linear Time-Varying Systems with Parameter Perturbations. IEEE Transactions on Automatic Control, Vol. 44, No. 10, pp.1919-pp. 1924.

De Souza, C.E., M. Fu and L. Xie (1993). $H_{\infty}$ Analysis and Synthesis of Discrete-Time Systems with Time-Varying Uncertainty. IEEE Transactions on Automatic Control, Vol. 38, No. 3, pp.459-462.

Garcia, G., J.B. Bernussou and D. Arzelier (1994). Robust Stabilization of Discrete-time Linear Systems with norm-bounded time-varying uncertainty. Systems \& Control Letters, Vol. 22, pp.327-pp.339.

Moheimani, S.O.R., A.V. Savkin and I.R. Petersen (1996). Necessary and sufficient conditions for robust observability of a class of discrete-time uncertain systems. Proceedings of the $35^{\text {th }} C D C$, Kobe Japan.

Xie, L., C.E. De Souza and M. Fu (1991). $H_{\infty}$ Estimation For Discrete-Time Linear Uncertain Systems. International Journal of Robust and Nonlinear Control, Vol. 1, pp.111-123.

Xie, L., Y.C. Soh and C.E. De Souza (1994). Robust Kalman Filtering for Uncertain Discrete-Time Systems. IEEE Transactions on Automatic Control, Vol. 39, No. 6, pp.1310-1314. 